\title{
Social Isolation Stress Provokes Atherosclerosis Through HPA-axis Over-Activation, Toll- Like Receptors, and Macrophage Polarization
}

\section{Arvin Haj-Mirzaian}

Tehran University of Medical Sciences

Kiana Ramezanzadeh

Tehran University of Medical Sciences

Armin Tafazolimoghadam

Tehran University of Medical Sciences

Mahla Radmard

Tehran University of Medical Sciences

Alireza Rahbar

Tehran University of Medical Sciences

Fardad Pirri

Tehran University of Medical Sciences

Ayda Khosravi

Tehran University of Medical Sciences

Niloufar Shababi

Tehran University of Medical Sciences

Ahmad Reza Dehpour ( $\nabla$ dehpoura@sina.tums.ac.ir)

Tehran University of Medical Sciences

Siavash Shariatzadeh

Tehran University of Medical Sciences

Michael Tajik

Tehran University of Medical Sciences

Farima Khalafi

Tehran University of Medical Sciences

Kiarash Kazemi

Tehran University of Medical Sciences

\section{Research Article}

Keywords: Social isolation stress, Toll-like receptor, Cardiovascular diseases, Atherosclerosis, M1 macrophage, HPA-axis 
Posted Date: July 20th, 2021

DOI: https://doi.org/10.21203/rs.3.rs-690532/v1

License: (c) (i) This work is licensed under a Creative Commons Attribution 4.0 International License. Read Full License 


\section{Abstract}

Background: It has been well documented that social isolation stress (SIS) can accelerate the formation of atherosclerotic plaque through inflammation. In this regard, this study aimed to elucidate the relation between HPA-axis, toll-like receptors (TLRs), as well as M1/M2 macrophage ratio and atherosclerosis in socially isolated $(\mathrm{SI})$ animals.

Methods: We used small interfering RNA (siRNA) against the glucocorticoid receptor (GR) to evaluate the relation between HPA-axis and TLRs. RT-PCR, ELISA, flow cytometry, and immunohistochemistry were used to assess any relation between atherosclerosis and TLRs, M1/M2 ratio, pro-inflammatory cytokines, and cell adhesion molecules in aortic tissue. Finally, we used TAK-242 (0.3 mg/kg, intraperitoneally), a selective antagonist of TLR4, as a possible prophylactic agent in formation of atherosclerotic plaque invivo.

Results: We observed that SI animals had higher serum concentration of corticosterone and higher body weight in comparison to normal animals. In SI animals, results of in-vitro study showed that knockingdown of the GR in bone marrow-derived monocytes significantly decreased the expression of TLR4. Invivo study suggested higher expression of TLR4 on circulating monocytes and higher M1/M2 ratio in aortic samples. Pathological study showed a mild formation of pre-atherosclerotic changes in SI animals. Finally, we observed that treating animals with TAK-242 could significantly inhibit the formation of preatherosclerotic changes.

Conclusion: SIS can possibly increase the risk of atherosclerosis through over-activating HPA-axis and subsequently led to TLR4 up-regulation, tissue inflammation, high M1/M2 ratio in endothelium. Thus, TLR4 inhibitors might be a novel treatment to reduce the risk of atherosclerosis-induced by chronic stress.

\section{Introduction}

There is a vast body of evidence indicated that psychological stress, such as social isolation stress (SIS), can be counted as a risk factor of cardiovascular diseases (CVDs) and might increase the risk of morbidity and mortality in CVDs cases [1-4]. Atherosclerosis is the main pathological basis of acute ischemic cardio-cerebrovascular events such as acute coronary syndrome (ACS), myocardial infarction (MI), and stroke [5]. Previously, it has been shown that chronic stress can promote the formation of atherosclerosis through excessive activation of the sympathetic nervous system, the renin-angiotensin system, oxidative stress, inflammation, and the hypothalamic-pituitary-adrenal cortex (HPA) axis [6-11].

It has been well documented that socially isolated animals had higher serum concentration of corticosteroids due to over-activation of HPA-axis $[10,11]$. Since glucocorticoid receptors (GR) agonists such as dexamethasone could suppress inflammation through inhibiting the nuclear factor-KB (NF-KB) $[12,13]$, current study hypothesized that HPA-axis might be linkage between SIS, innate immune system, and atherosclerosis. 
Extensive uptake of lipoprotein and modified lipoprotein, including oxidized low-density lipoprotein (ox$\mathrm{LDL}$ ) by differentiated macrophages (derived from circulating monocytes into endothelium by interactions with their cell adhesion receptors, including P-selectin, E-selectin, intercellular adhesion molecule-1 (ICAM-1), and vascular cell adhesion molecule-1 (VCAM-1)) might result in the formation of lipid-laden macrophage $[14,15]$.

On the other hand, two major subtypes of macrophages including M1 and M2 macrophages have been strongly linked to the formation of atherosclerosis plaque [16]. It has been demonstrated that M1 macrophages are the most prevalent macrophage in atherosclerosis plaque [16, 17]. In addition, it is postulated that polarization of M2 phenotype has an anti-atherosclerotic effect [18]. Interestingly, the polarization of macrophages into M1 or M2 phenotypes has been associated with inflammation and tolllike receptors (TLRs) 2 and 4 signaling pathway [19-21].

TLRs, as a part of the innate immune system, are the most characteristic members of pattern recognition receptors (PRRs) [22]. Activation of TLRs by various ligands such as pathogen-associated molecular patterns (PAMPs) might result in increased expression of inflammatory cytokines [22]. A few couples of studies have shown that inappropriate activation of TLRs might play a key role in the pathogenesis of atherosclerosis [23]. However, the sub-stream pathway of the atherosclerosis and excessive activation of TLRs is still unclear.

Previous studies have used genetically modified animals (ApoE knock out mice) to evaluate the role of chronic stress on atherosclerotic plaque formation; however, this non-physiological model could not accurately reflect the human disease. For example, it has been suggested that ApoE knock out mice are expected to have higher levels of very-low-density lipoprotein (VLDL), which is not typical in human atherosclerosis [7]. Therefore, in this study, we not only focus on the pathophysiology of atherosclerosis, but also, introduce a new novel model, which could reflect human pre-atherosclerosis pathophysiology more accurate than genetically modified animals.

Since the underlying mechanisms of SIS-induced atherosclerosis is yet to be established, this study was aimed to elucidate the effect of SIS on the formation of atherosclerotic plaque in normal mice with high fat diet through M1 and M2 macrophage polarization and evaluate the role of TLR4 signaling pathway and HPA-axis in the recently mentioned subject.

\section{Material And Methods}

\subsection{Animals and housing conditions}

This study used a total of 30 male C57BL/6J mice aged $21-25$ days and weighing $20-22 \mathrm{~g}$. Animals were held in two separate conditions: social condition (SC) and isolated condition (IC). The standard laboratory conditions were applied for all animals for a period of 5 weeks (the temperature was $22 \pm 2{ }^{\circ} \mathrm{C}$, the humidity was $50 \pm 10 \%$, the lights were 12-hour dark and free access to food and water). Socially conditioned mice were placed in Plexiglas boxes $(25 \mathrm{~cm} \times 25 \mathrm{~cm} \times 15 \mathrm{~cm})(6$ mice per cage $)$ and IC 
animals were placed individually in Plexiglas boxes $(24 \mathrm{~cm} \times 17 \mathrm{~cm} \times 12 \mathrm{~cm})[24,25]$. In addition, both groups including SC and IC were fed with high-fat diet (HFD) containing $21 \%$ saturated fat and $0.15 \%$ cholesterol. The same experimenter washed the cages of IC animals regularly to minimize care and social contact. Socially conditioned animals were considered as control group or normal animals in in-vivo studies.

\subsection{Experimental design and sample acquisition}

In this study, we evaluate the impact of SIS on atherosclerosis process at both level of in-vitro and in-vivo. At in-vitro level, we cultured the bone marrow-derived monocyte, which have been obtained from socially isolated animals; then we evaluated the gene expression of TLR2 and TLR4 after knocking-down the GR or NF-KB in medium contained dexamethasone.

At in-vivo level, IC animals were divided into two groups prior to a 5-week period of isolation (IC animals and IC animals treated with TAK-242). TAK-242, a selective TLR4 inhibitor, was dissolved in in 1\% Dimethyl sulfoxide (DMSO) and then diluted in sterile water [26]. TAK-242 (purchased from Sigma Aldrich Co., LLC) was administrated at a dose of $0.3 \mathrm{mg} / \mathrm{kg}$ intraperitoneal twice a week for consecutive 5 weeks of isolation period in IC animals based on previous studies [10,24].

After a 5-week period of isolation, mice were prepared for the experimental tasks such as electrocardiography assessment. In the next step, the cardiac blood samples were obtained and entire aorta was resected and immediately sent for further analysis.

\subsection{Electrocardiography}

For evaluating the cardiac function, we assessed the echocardiography and electrocardiogram (ECG). In this regard, both isolated and normal mice were anesthetized using $2 \%$ isoflurane in oxygen and maintained at body temperature. ECG was recorded in normal animals and after the 5-week period of isolation in socially isolated animals. ECG was recorded for $15 \mathrm{~min}$ in mice. Needle electrodes attached to a bio-amplifier (ADI Devices, Spain) were implanted subcutaneously for limb lead at position II. The QT interval, QRS complex, and ST segments were assessed for each ECG tracing. The signals were digitized by a Powerlab system at a sampling rate of $10 \mathrm{kHz}$, and displayed using the software Lab Map 7 (ADInstrument, Australia). The QT intervals, presented as corrected QT (QTC), were calculated in a 5 min ECG. The QTc was obtained using Bazett's formula $(\mathrm{QTC}=\mathrm{QT} / \sqrt{ }(\mathrm{RR} / 100))$

\subsection{Assessment of lipid profile}

Total cholesterol, triglycerides (TG), low-density lipoprotein (LDL), high-density lipoprotein levels (HDL) were measured in plasma samples according to manufacturer instructions from Infinity Triglycerides and Wako Chemicals. In brief, plasma samples were mixed with equal volumes of dextran sulfate $(20 \mathrm{~g} / \mathrm{L})$ and magnesium chloride $(1 \mathrm{M})$ solution $\mathrm{pH} 7.0$ and incubated at room temperature for 10 minutes followed by centrifugation at $4{ }^{\circ} \mathrm{C}$ for 30 minutes. The supernatant was calculated by means of chromatography to determine the concentration of total cholesterol, free cholesterol, triglycerides, low density lipoprotein (LDL), and high-density lipoprotein cholesterol. 


\subsection{Assessment of inflammatory cytokines}

In this experiment we applied the following measures to assess the molecular assay in target tissue. In this step, after euthanasia, we separated longitudinal axis of thoracic and iliac aorta from the aorta root and the junction of heart. Entire aorta including aortic sinuses, aortic arch, thoracic aorta, abdominal aorta were isolated and homogenized based on our previous studies after animal euthanasia of each experimental community [27-29]. The mixed venous-arterial blood was collected in heparinized containers and centrifuged to measure circulating cytokine levels. Pooled plasma from both IC and SC groups was stored at -80 degrees $C$ until cytokine concentrations were assessed. Samples were centrifuged at $1,466 \times \mathrm{g}$ for $15 \mathrm{~min}$ at $4{ }^{\circ} \mathrm{C}$ after the samples were homogenized [30]. The TNF- $a$, IL-6, and IL-1 $\beta$ levels were calculated using enzyme-linked immunosorbent assay kit (ELISA; Abcam, Cambridge, MA, USA). The amounts of TNFa, IL-6, and IL-1 $\beta$ were calculated according to instructions from the supplier by using a standard curve.

\subsection{Real-time PCR analysis}

Aorta was resected and immediately frozen by using liquid nitrogen, and stored at $-80^{\circ} \mathrm{C}$. In the first step total RNA was extracted using Trizol reagent (Invitrogen, Cergy Pontoise, France) from the entire aorta. Gene-level alterations in mRNA were calculated using qRT-PCR after the reverse transcription of 1 $\mu \mathrm{g}$ of RNA from each sample using PrimeScript RT reagent kit (Takara Bio Inc., Otsu, Japan). By using SYBR Premix Ex Taq Technology (Takara Bio), qRT-PCR was completed on a light cycler device (Roche Diagnostics, Mannheim, Germany). Primer sequences have been designed based on previous reports, and are shown in Table 1. Thermal cycling conditions included an initial activation step for $30 \mathrm{~s}$ at $95^{\circ} \mathrm{C}$ afterwards 45 cycles as well as a denaturation step for $5 \mathrm{~s}$ at $95^{\circ} \mathrm{C}$ and a combined annealing/extension step for $20 \mathrm{~s}$ at $60^{\circ} \mathrm{C}$ [25]. Analysis of the melting curve was conducted to certify if all primers yielded a single PCR product. 
Table 1

Sequences of primers used for quantitative RT-PCR in mice

\begin{tabular}{|ll|}
\hline Gene name & Primers (forward and reverse 5' to 3') \\
\hline ICAM-1 & F: 5'-TTCACACTGAATGCCAGCTC-3' \\
& R: 5'-GTCTGCTGAGACCCCTCTTG-3' \\
\hline P-selectin & F: 5'-CCCAGGTGGAGGTCTACTCA-3' \\
& R: 5'-CAGGATTTTGGGAGCTGGTA-3' \\
\hline E-selectin & F: 5'-AAGTGGACGGGAGAGAGTTTGGTGT-3' \\
& R: 5'-ACGCAAGTTCTCCAGCTGTT-3' \\
\hline TLR2 & F: 5'-CAGCTGGAGAACTCTGACCC-3' \\
& R: 5'-CAAAGAGCCTGAAGTGGGAG-3' \\
\hline TLR4 & F: 5'-CAACATCATCCAGGAAGGC-3' \\
& R: 5'-GAAGGCGATACAATTCCACC-3' \\
\hline NF-KB & F: 5'-CTGGTGGACACATACAGGAAGAC-3' \\
& R: 5'-ATAGGCACTGTCTTCTTTCACCTC-3' \\
\hline
\end{tabular}

\subsection{Immunohistochemistry (IHC)}

Immunohistochemistry (IHC) has evaluated the expression of macrophage surface receptors in entire aorta samples, including CD163 (M2 marker) and CD86 (M1 marker). Samples of entire aorta have been resected and washed in 4 steps with 5 min intervals by PBS. In order to retrieve the antigens, sections were then put in 2 normal hydrochloric acid (HCL) solutions for 30 min. In the next step, adding Borate buffer for 5 min neutralized the HCL effect. Samples were then washed with PBS and then a 3\% Triton X100 solution was used for 30 minutes to permeate the cell membranes, and PBS was again provided for sample cleaning. Goat serum (10,0\%) was applied in 30 minutes to suppress the secondary antibody reaction as an extra color to the background. The samples were then added a primary antibody diluted with PBS (1:100), and the mixture was kept in a refrigerator $\left(2-8^{\circ} \mathrm{C}\right)$ for one day. Then a secondary antibody was added which was diluted with PBS (1:150). The samples were incubated in a $37^{\circ} \mathrm{C}$ incubator for one and a half hours. Afterward, 4',6-diamidino-2-pheny-lindole (DAPI) were added in a dark room and PBS was poured on the samples immediately. Finally, for the cell measurement, the samples were divided into five different areas by using fluorescent Olympus microscope (TET400). Images were analyzed using the ImageJ (Fiji version) software in the next step. Details were obtained of the proportion 
of positive immunolabeled cells in the total cells in each selected region (the ratio number of positively stained cells/total number of cells $\times 100$ ).

\subsection{Flow cytometry}

The cardiac blood samples were obtained in heparinized tubes for further study after animal euthanasia in all our experimental classes. The samples were then centrifuged at $500 \times \mathrm{g}$ at $4^{\circ} \mathrm{C}$ for 10 minutes, and the supernatants were discarded. The cells were finally resuspended into an RBC buffer of $3 \mathrm{ml}$ (for every mouse) and incubated at room temperature for 10 minutes and washed cells by adding a $47 \mathrm{ml}$ of medium RPMI 1640 and 2\% FBS. The cell pellet resuspended with $0.5 \mathrm{ml}$ of RPMI 1640 medium and $2 \%$ FBS after centrifuging the samples at $500 \times \mathrm{g}$ for $10 \mathrm{~min}$ at $4^{\circ} \mathrm{C}$ and discarding the supernatant [31]. The cells were finally resuspended in $1 \mathrm{~mL}$ of PBS, which contained $2 \%$ FBS. All samples were eventually stained with antibodies, as mentioned below.

In the next step, all nucleated cells, which were obtained from the previous step were subsequently stained for CD11b clone M1/70 PE-Cy7 (2 ng/ul; eBioscience Ltd., UK) and Ly6G clone 1A8 APC (2 ng/ul; eBioscience Ltd., UK) for 30 min in medium containing $2 \%$ FBS for sorting monocytes. Purified and sorted monocytes were evaluated for the expression of TLR2 and 4 by using TLR4 antibody (HTA125, mouse IgG2a) and TLR2 (TL2.1, mouse IgG2a) as well as corresponding isotype antibodies (purchased from eBioscience, San Diego, CA). Cells were then washed after staining with the above-mentioned antibodies, resuspended in RPMI-medium with $2 \%$ FBS, and analyzed by using the BD FACSCaliburTM flow cytometer tool and FlowJo software (TreeStar inc.).

\subsection{Histopathological study}

For histopathological examination, entire aorta sections were stained with hematoxylin and eosin (H\&E). The staining process was carried out according to the protocol below: deparaffinized of the specimens were done at $70^{\circ} \mathrm{C}$ for $20 \mathrm{~min}$ by using xylene solution, and then rehydrated in two changes of absolute alcohol. Slides is rinsed in hematoxylin solution for $15 \mathrm{~min}$ to stain the nuclei, and then washed for $5 \mathrm{~min}$ in running tap water. After bluing in $0.2 \%$ ammonia water and then washing in tap water for $5 \mathrm{~min}$, the slides were counterstained in eosinphloxine for $1 \mathrm{~min}$ and then samples were rinsed in $90 \%, 96 \%$ and $100 \%$ ethanol for $2 \mathrm{~min}$. Slides were then embedded in both xylene and mounting media. Eventually, samples were used to monitor inflammation of the tissue and atherosclerotic lesions using an optical microscope (resolution: 40x).

\subsection{Assessment of corticosteroid concentration, body weight and organs weight}

To evaluate the basal corticosterone levels, blood sample acquisition was done according to previously published methods $(1,2)$. Briefly, both socially isolated and normal mice under their basal conditions were decapitated under $\mathrm{CO}_{2}$ anesthesia (all procedures were done at $9 \mathrm{AM}$ ); then trunk blood samples were collected for further analysis $[10,11]$. Then, all samples were centrifuged at $3000 \cdot \mathrm{g}$ for $10 \mathrm{~min}\left(4^{\circ}\right.$ C); then the serum samples obtained from previous step were stored at $20^{\circ} \mathrm{C}$ until the assay day. 
Corticosterone levels were measured using a commercial ELISA kit (Biospes, China). In addition, body weight $(\mathrm{g})$ and organs including adrenals $(\mathrm{mg})$ and thymus $(\mathrm{mg})$ were removed and weighted in both socially isolated and normal animals.

\subsection{Isolation and culture of monocytes for in-vitro study}

Based on our previous study, after sacrificing mice, we resected femur and tibia from surrounding tissue of both normal and socially isolated animals, and then flush the bones with warm medium ( $5 \%$ fetal bovine serum (FBS), + 1\% Streptomycin) through a $70 \mu \mathrm{m}$ nylon [32]. Then centrifuged the samples $250 \mathrm{x}$ $\mathrm{g}$ for $10 \mathrm{~min}$ in $4^{\circ} \mathrm{C}$; and discarded the supernatant and resuspend the cells with approximately $25 \mathrm{ml}$ of medium [33]. We checked and counted the cells in a counting chamber with a light microscope and calculated the number of observed cells. Then we prepared cell for culture, in this regard we seed the cells on 6-well ultra-low-attachment surface plates to prevent permanent adhesion to the bottom of the plate. Use a concentration of 106 cells per $\mathrm{ml}$ with up to $6 \mathrm{ml}$ per well and $20 \mathrm{ng} / \mathrm{ml} \mathrm{M-CSF}$ was used to promote cell differentiation; and culture the cells for at least 5 days at $37^{\circ} \mathrm{C}$ in $5 \% \mathrm{CO}_{2}$. All cells were evaluating to be CD11b positive by using flowcytometry.

\subsection{Inhibiting the expression of GR and NF-KB in cultured monocytes using siRNA}

In this study, we used siRNA against GR and NF-KB to inhibit the expression of GR in cultured monocytes. Both negative control (non-silencing random siRNA) and siRNA against GR and NF-KB were purchased from Ambion (Invitrogen, Carlsbad, CA) [34]. During the culturing process in 6-well plates, siRNAs transfected with the concentration of $15 \mathrm{nM}$ using PepMute Plus siRNA transfection reagent (SignaGen Laboratories, ljamsville, MD), based on the manufacturer's instruction. Samples were divided into three groups as follow: 1- cells transfected negative control siRNA, 2- cells transfected siRNA against GR, and, 3- cells transfected siRNA against NF-KB. Cells were allowed to recover for a week in medium with adding $0.01 \mu \mathrm{mol} / \mathrm{L}$ of dexamethasone [34]. After one week of incubation, samples were evaluated for gene expression of NF-KB, TLR2, and TLR4 by using RT-PCR as mentioned above.

\subsection{Statistical analysis}

In the current study, One-Way ANOVA and T-test analysis were used to analyze the data by using SPPSS version 25 and graphs were designed by GraphPad Prism version 8. P value $<0.05$ was as a statistical significance. In addition, the sample size was determined using version 3 of G*Power software, considering the study's power of 0.8 and $a=0.05$.

\subsection{Ethics}

Our study was in accordance with the National Institute of Health $(\mathrm{NIH})$ Guidelines for the Care and Use of Laboratory Animals (HHS publication $85-23,1985$ ), legislation for the protection of animals used for scientific purposes (Directive 2010/63/EU) and also this study was carried out in compliance with the 
ARRIVE guidelines (PLoS Bio 8(6), e1000412,2010). All experimental protocols were approved by a Tehran University of Medical Sciences (TUMS) Ethics committee and according to TUMS policies on medical and research ethics (Code: IR.TUMS.VCR.REC.1397.316). Euthanasia was done by using 7 minutes exposure to $\mathrm{CO} 2$ and followed by cervical dislocation for confirmation of death.

\section{Results}

\subsection{Effect of social isolation stress on serum concentration of corticosterone}

In this section, we evaluated the serum concentration of corticosterone, body weight, and organs weight including adrenal and thymus in both normal and socially isolated animals (Table 2). T-test analysis showed that socially isolated animals had significantly higher level of corticosterone $(42.30 \pm 7.67 \mathrm{ng} / \mathrm{ml})$ in comparison to normal animals $(33.30 \pm 5.03 \mathrm{ng} / \mathrm{ml}, \mathrm{t}=3.101$, $\mathrm{df}=18$, and $P<0.01)$. Also, we evaluated that the body weight before and after four weeks of experiment in both socially isolated and normal animals. We observed no significant difference between these two groups of mice before starting the experiment animal (IC: $20.32 \pm 0.71, \mathrm{SC}: 20.58 \pm 0.51, \mathrm{t}=0.940, \mathrm{df}=18$, and $P>0.05$ ). After four weeks, the mean weight observed in socially isolated animals $(27.08 \pm 1.6 \mathrm{~g})$ was significantly higher than normal animals $(25.56 \pm 1.1 \mathrm{~g}, \mathrm{t}=2.476 \mathrm{df}=18$, and $P<0.05)$. On the other hand, we did not observed any significant difference in adrenal (IC: $3.51 \pm 0.14$, SC: $3.49 \pm 0.12$ ) and thymus (IC: $57.12 \pm 2.8$, SC: $58.31 \pm 2.6$ ) weights in these two groups $(\mathrm{t}=0.343 \mathrm{df}=18$, and $P>0.05 ; \mathrm{t}=0.984, \mathrm{df}=18$, and $P>0.05$, respectively).

Table 2

Absolute body weight (g) and organ weights ( $\mathrm{mg}$ ) in both socially isolated (IC) and normal mice (SC).

\begin{tabular}{|c|c|c|c|c|c|}
\hline Items & $\begin{array}{l}\text { Concentration of corticosterone } \\
(\mathrm{ng} / \mathrm{ml} n=10)\end{array}$ & $\begin{array}{l}\text { Body weight } \\
\text { before } \\
(g, n=10)\end{array}$ & $\begin{array}{l}\text { Body weight } \\
\text { after } \\
(g, n=10)\end{array}$ & $\begin{array}{l}\text { Adrenals } \\
(\mathrm{mg}, n= \\
10)\end{array}$ & $\begin{array}{l}\text { Thymus } \\
\text { (mg, } n= \\
10 \text { ) }\end{array}$ \\
\hline IC & $42.3 \pm 7.7^{\star *}$ & $20.32 \pm 0.71$ & $27.08 \pm 1.6^{*}$ & $3.51 \pm 0.14$ & $57.12 \pm 2.8$ \\
\hline SC & $33.3 \pm 5.0$ & $20.58 \pm 0.51$ & $25.56 \pm 1.1$ & $3.49 \pm 0.12$ & $58.31 \pm 2.2$ \\
\hline
\end{tabular}

\subsection{Assessment the role of corticosteroids on expression of NF-KB, TLR2, and TLR4}

In order to clarify that over-activation of GR (in consequence of high serum corticosterone) could impact on the expression of TLR2 and TLR4 on monocytes, we used cultured monocytes derived from both normal and socially isolated animals' bone marrow. In this section, we hypothesized that over-activation of GR in socially isolation animals could affect the expression level of TLRs through NF-KB signaling 
pathway. In this regard, we knock-down the expression of GR and NF-KB by using siRNA in cultured monocytes. It should be noted that we checked and verified the inhibitory effects of GR and NF-KB siRNAs prior to performing any further investigation (Fig. 1A). T-test analysis showed that both GR-siRNA and NFKB-siRNA could significantly knock-down the expression of GR and NF-KB $(\mathrm{t}=24.87, \mathrm{df}=8$, and $P<0.001$, and $\mathrm{t}=47.33$, $\mathrm{df}=8$, and $P<0.001$, respectively).

In this section, we used GR and NF-KB siRNAs in only cultured cells extracted from socially isolated animals' bone marrow, because using GR and NF-KB siRNAs in cultured cells extracted from normal animals' bone marrow could not change the justification. One-Way ANOVA analysis showed that GR and NF-KB knocking-down could significantly alter the expression of TLR4 and TLR2 $(F(15,64)=71.94, P<$ 0.001 , Fig. 1B). Post hoc analysis showed that cultured monocytes derived from socially isolated animals had significantly higher expression for TLR4, TLR2, and NF-KB in comparison to normal animals $(\mathrm{P}<$ 0.001). Also, the expression of both TLR4 and NF-KB significantly dropped after knocking-down the GR in the medium contained with $0.01 \mu \mathrm{mol} / \mathrm{L}$ dexamethasone in cultured monocytes derived from socially isolated animals' bone marrow $(P<0.001)$. The expression of TLR4 decreased significantly after knocking-down the NF-kB in comparison to cell treated with GR-siRNA in the medium contained with $0.01 \mu \mathrm{mol} / \mathrm{L}$ dexamethasone $(P<0.001)$. In addition, in comparison to cultured monocytes derived from socially isolated animals' bone marrow, the expression level of TLR2 remained intact after treating cells with GR-siRNA $(P>0.05)$, however, the expression level of TLR2 significantly decreased after treating cells with NF-KB-siRNA in comparison to cultured monocytes derived from socially isolated animals' bone marrow $(P<0.05)$. On the other hand, our results showed that $G R$-siRNA could significantly decrease the expression of NF-KB, TLR2, and TLR4 in cultured monocytes derived from normal animals' bone marrow in comparison to cells treated with vector $(P<0.05)$; also, results of NF-KB knocking down showed significant down-regulation of both TLR4 and TLR4 in cultured monocytes derived from normal animals' bone marrow in comparison to cells treated with vector $(P<0.001)$. These results showed a tight interaction between GR, NF-KB, and TLR4 in cultured monocytes derived from both normal and socially isolated animals' bone marrow. Our in-vitro study showed that over-activation HPA axis and consequently over-activation of GR in socially isolated animals could result in up-regulation of NF-KB, TLR4 and TLR2.

\subsection{Assessment of plasma level of lipid profile}

In the first step of the current study, we assessed the lipid profile in socially isolated animals, as well as normal mice. One-Way ANOVA analysis showed that social isolation significantly impacted on the plasma concentration of HDL, LDL, TG, and cholesterol in comparison to the control group $(F(15,144)=$ 224.1, $P<0.001$, Fig. 2). Tukey's test showed that socially isolated animals had significantly higher plasma levels of LDL, TG, and cholesterol $(P<0.01, P<0.001, P<0.01$, respectively), in addition to significant lower plasma concentration of $\mathrm{HDL}$ in comparison to the control group $(P<0.05)$. However, post hoc test showed that blockage of TLR4 by using administration of TAK-242 at a dose of $0.3 \mathrm{mg} / \mathrm{kg}$ (twice a week) could not significantly alter the impact of SIS on plasma concentration of lipid profile components in socially isolation animal $(P>0.05)$. Also, our results demonstrated that the proportion of LDL to HDL was significantly higher in both IC $(2.82 \pm 0.31)$ and IC animals treated with TAK-242 (3.12 \pm 
$0.56)$ in comparison to SC mice $(1.39 \pm 0.22, P<0.001)$. However, no significant difference was observed between IC and IC animals treated with TAK-242 ( $P>0.05)$; and also, no significant effect was observed in normal animals treated with TAK-242 $(P>0.05)$.

\section{4 Evaluation the effect of SIS on expression of TLR2 and TLR4 on the surface of circulation monocytes}

In this step we tried to evaluate the impact of SIS on the expression of TLR2 and 4 on circulating monocytes by using blood samples. Data obtained from flowcytometry showed that social isolation stress significantly increased the expression of TLR4 on circulating monocytes $(\mathrm{t}=0.99, \mathrm{df}=8, P<0.001$, Fig. 3). However, t-test analysis failed to show any significant difference between the expression of TLR2 in social condition and isolation condition animals $(t=8.11$, $\mathrm{df}=8, P>0.05$, Fig. 3 ).

\subsection{Evaluation of the leukocyte adhesion receptors gene expression}

Results obtained from One-Way ANOVA analysis showed a significant difference between IC animals, socially isolated animals treated with TAK-242, and SC animals in gene expression of VCAM-1, ICAM-1, Eselectin, and P-selectin receptors in entire aorta samples $(F(15,144)=49.63, P<0.001$, Fig. 4). Tukey's analysis showed a significantly higher fold change in gene expression VCAM-1, ICAM-1, E-selectin, and Pselectin in IC animals in comparison to SC group $(P<0.001)$. On the other hand, socially isolated animals that were treated with administration of TAK-242 at $0.3 \mathrm{mg} / \mathrm{kg}$ showed non-significant changes in gene expression of ICAM-1, E-selectin, and P-selectin in comparison to SC animals $(P>0.05)$. In addition, we observed socially isolated animals treated with TAK-242 had significantly lower gene expression of VCAM-1 $(P<0.001)$, ICAM-1 $(P<0.05)$, E-selectin $(P<0.001)$, P-selectin $(P<0.001$ in comparison IC animals. Besides, we did not observe any significant changes after administration of TAK-242 in normal animals.

\subsection{Evaluation the effect of SIS on macrophage polarization}

In the next step, we tried to evaluate the polarization of monocyte-derived macrophages by using immunohistochemistry. One-Way ANOVA analysis showed that SIS significantly impacted on the proportion of $M 1$ to $M 2$ macrophages biomarkers expression $(F(3,44)=126.0, P<0.001$, Fig. 5$)$. Tukey's test demonstrated that the SIS significantly increased the percentage of macrophages positive for the M2 marker (CD163, $P<0.001)$ in comparison to SC animals. Whereas, administration of TAK-242 twice a week $(0.3 \mathrm{mg} / \mathrm{kg})$ in socially isolated animals remarkably decreased the $\mathrm{M} 1 / \mathrm{M} 2$ ratio in comparison to IC animals $(P<0.001)$, however, no significant effect was observed in normal mice treated with TAK-242 ( $P$ $<0.05)$. In addition, post hoc test showed that socially isolated animals treated with TAK-242 had a nonsignificant M1/M2 ratio difference with normal animals treated with vehicle $(P>0.05)$.

\subsection{Assessment of M1 and M2 sub-stream biomarkers}


One-Way ANOVA analysis demonstrated that SIS and the blockage of TLR4 could significantly alter the level of TNF-a $(\mathrm{F}(3,36)=98.61, P<0.001$, Fig. 6), IL-1b $(\mathrm{F}(3,36)=48.19, P<0.001$, Fig. 6$)$, and IL-6 (F (3, $36)=27.1, P<0.001$, Fig. 6) in entire aorta samples in comparison to SC animals. Tukey's test showed that SIS significantly increased the concentration of TNF-a in entire aorta specimens in comparison to SC animals $(P<0.001)$. Although, post hoc analysis showed that the mean concentration of TNF-a in entire aorta samples was significantly higher in socially isolated animals treated with administration of TAK242 in comparison to SC mice $(P<0.001)$, treating IC animals with injection of TAK-242 significantly decreased the concentration of TNF-a in comparison to IC mice $(P<0.001)$. We observed the same results in the evaluation of IL-1b and IL-6. Our results showed that SIS significantly increased the level of IL-1b in entire aorta samples in comparison to SC animals $(P<0.001)$; TAK-242 treatment decreased the level of $\mathrm{IL}-1 \mathrm{~b}$ in comparison to IC animals $(P<0.001)$. On the other hand, we observed a significantly higher level of IL-6 in entire aorta samples of IC animals in comparison to SC animals $(P<0.001)$, whereas IC animals treated with TAK-242 had a significantly lower concentration of IL-6 in entire aorta samples in comparison to IC mice with no treatment $(P<0.001)$.

\subsection{Aorta histopathological study}

A total of 40 entire aorta specimens from 40 animals, including 10 mice in each group were assessed for pathological study (Fig. 7). Our results showed a significant difference in the presence of preatherosclerotic intimal lesions and pathologic intimal thickening between IC, SC, and IC animals treated with TAK-242. In this regard, One-Way ANOVA analysis showed that intima/media ratio was significantly higher in socially isolated animals in comparison to normal animals $(F(3,36)=5.950, P<0.001, F i g .7)$. Tukey's analysis demonstrated that administration TAK-242 could significantly inhibit the intimal thickening and decrease intima/media ratio in socially isolated animals $(P<0.05)$. However, no significant effect was observed after treating normal animals with TAK-242 on intima/media ratio $(P>$ 0.05). In addition, we observed that IC animals treated with TAK-242 had significantly lower pathological lesions in comparison to IC animals. Although TAK-242 was significantly inhibited the intimal thickening, results obtained from histopathological study showed that IC animals treated with TAK-242 had a slightly higher presence of pathological lesions in comparison to SC animals. Among all samples in IC group, 7 (70\%) had pre-atherosclerotic intimal lesions, and 4 (40\%) had pathologic intimal thickening. In addition, among samples obtained from IC mice treated with TAK-242 at a dose of $0.3 \mathrm{mg} / \mathrm{kg}, 3$ samples (30\%) had pre-atherosclerotic intimal lesions, and no sample had significantly pathologic intimal thickening in comparison to animals treated with vehicle. It should be noted that we did not observe any direct and progressive pathological atherosclerotic lesion in all samples.

\subsection{Evaluation of electrocardiogram}

We have assessed the cardiac electrical function among all studied groups in this manuscript. One-Way ANOVA analysis showed that socially isolated animals had a significantly higher heart rate (HR) in comparison to SC animals $(F(3,36)=6.514, P<0.01$, Table 3$)$. However, One-Way ANOVA results failed to show any significant difference among $P Q$ interval duration $(F(3,36)=0.468, P>0.05$, Table 3$)$, corrected QT interval (QTc) duration $(\mathrm{F}(3,36)=0.975, P>0.05$, Table 3$)$, and QRS complex duration $(\mathrm{F}(3,36)=$ 
$0.152, P>0.05$, Table 3 ) in IC, SC, and IC animals treated with TAK-242. Post hoc tests showed that HR in IC and IC animals treated with TAK-242 had significantly higher in comparison to SC mice $(P<0.05)$; however, IC and IC animals treated with TAK-242 had no significant difference $(P>0.05)$. In addition, we did not observe any arrhythmia among all studied ECGs in all groups.

Table 3

ECG results of normal and socially isolated animals.

\begin{tabular}{|lllll|}
\hline Items & $\begin{array}{l}\text { Social } \\
\text { condition }(n=\end{array}$ & $\begin{array}{l}\text { Social condition + TAK- } \\
\text { 10) }\end{array}$ & $\begin{array}{l}\text { Isolation } \\
\text { condition }(n=10) \\
10)\end{array}$ & $\begin{array}{l}\text { Isolation condition + } \\
\text { TAK-242 }(n=10)\end{array}$ \\
\hline $\begin{array}{l}\mathrm{HR} \\
(\mathrm{bpm})\end{array}$ & $383 \pm 37$ & $377 \pm 33$ & $451 \pm 47^{*}$ & $443 \pm 68^{*}$ \\
\hline $\begin{array}{l}\mathrm{PQ} \\
\text { (msec) }\end{array}$ & $45.9 \pm 10.3$ & $48.6 \pm 9.9$ & $51.3 \pm 12.6$ & $47.8 \pm 8.1$ \\
\hline $\begin{array}{l}\text { QRS } \\
\text { (msec) }\end{array}$ & $10.9 \pm 0.7$ & $11.1 \pm 0.7$ & $11.1 \pm 1.8$ & $10.8 \pm 1.3$ \\
\hline $\begin{array}{l}\text { QTc } \\
\text { (msec) }\end{array}$ & $42.7 \pm 3.5$ & $42.3 \pm 2.2$ & $41.8 \pm 1.8$ & $40.8 \pm 2.7$ \\
\hline
\end{tabular}

HR: heart rate, bpm: beats per minute, msec: millisecond. ${ }^{*} P<0.05$ compared to social conditioned animals.

\section{Discussion}

In the current study, we examined the possible role of the TLRs, inflammation, and M1/M2 ratio in formation of atherosclerotic plaque in socially isolated animals. Our result showed that SIS could increase the risk of atherosclerosis by enhancing the plasma concentration of total cholesterol, TG, and LDL/HDL. In addition, we observed higher body weight gained in socially isolated animals in comparison to normal animals, but no significant difference was detected between organs weight, including adrenals and thymus, in socially isolated and normal mice. Higher expression of TLR4 on the surface of circulating monocytes in socially isolated animals was observed. Also, higher levels of pro-inflammatory cytokines including TNF-a, IL-1 $\beta$, and IL-6 in addition to higher M1/M2 were observed in entire aorta specimens of socially isolated animals. The high presence of pre-atherosclerotic lesions in addition to higher intima/media ratio in entire aorta samples obtained from socially isolated animals have confirmed that SIS could increase the risk of atherosclerosis. In addition, in-vitro study demonstrated that HPA-axis overactivation in socially isolated animal, might increase the expression of TLR4 through NF-KB pathway. Although TLR4 inhibitor (TAK-242) could not induce a significant impact on lipid profile components, TAK-242 significantly inhibited the atherosclerosis process at both pathological and molecular levels by decreasing the endothelium inflammation and M1/M2 ratio. Finally, the electrocardiographic study showed that SIS could significantly increase the heart rate, but no impact on cardiac electrical function. 
The impact of social isolation stress on the risk of CVDs might be mediated through stress-related dysregulation of cardiovascular, metabolic, and neuroendocrine processes [35]. In this regard, numerous studies have shown that SIS could significantly induce lipid dysregulation and suggested that social stress could alter lipid metabolism by HPA axis dysfunction and enhancing the transcriptional activity of genes involved in lipid synthesis [35,36]. Gądek-Michalska et al. showed chronic SIS could significantly increase both IL-1 $\beta$ and corticosterone [37]. On the other hand, previous studies demonstrated that corticosteroids suppress the immune response by inhibiting the NF-KB [38]. Although, it has been suggested that the expression of NF-KB can be regulated by TLRs activity, the reverse interaction between them has not been clearly reported. In this study, we observed that SIS increased the serum concentration of corticosterone in-vivo; and high level of corticosterone might result in inhibition of NF-kB and upregulation of TLR4 as the first part of the puzzle. Interestingly, upregulation in NF-KB, TLR2, and TLR4 in daughter cells might suggest a possible epigenetic change in bone marrow-derived monocyte from socially isolated animals. Therefore, further investigation must be conducted to evaluate the role of epigenetic changes in atherosclerosis induced by social isolation stress.

It has been proposed that the levels of total cholesterol, TG, and LDL were markedly higher in animals exposed to chronic unpredictable stress (CUS) [29]. In line with the previous evidence, our results showed that SIS increased the plasma concertation of total cholesterol, and TG, and LDL, as well as increasing LDL/HDL ratio. In addition, previous studies have shown that animals exposed to chronic stressor such as SIS and maternal separation were predisposed to CVDs through mitochondrial dysfunction and increasing cardiac oxidative stress $[39,40]$. Since ox-LDL plays a key role in the formation of atherosclerosis [41], a combination of high LDL and oxidative stress levels in vascular tissue could cause an increment in ox-LDL level and subsequently increase the risk of atherosclerosis. This evidence would be the second part of the puzzle.

It has been well documented that oxidative stress could promote vascular inflammation [42]. In addition, inflammatory response to oxidative stress and increase in the pro-inflammatory cytokines, including TNFa, IL-1 $\beta$, and IL-6, has been associated with increasing adhesion molecule expression such as ICAM- 1 and VCAM-1 [43]. Increase in the expression of these adhesion molecules could increase the rate of monocyte trafficking across the vascular wall [44]. Previous studies showed that social disruption stress led to release of the pro-inflammatory cytokine and increased inflammation in endothelium [28]. In addition, high expression of ICAM-1 and VCAM-1 was observed in CUS [29]. In consistent with previous reports, current study showed that SIS increased the endothelium inflammation by enhancing the level of TNF-a, IL-1 $\beta$, and IL-6. Besides, we showed that the gene expression of adhesion molecules, including ICAM- 1 , VCAM-1, E-selectin, P-selectin was significantly increased following SIS. Taking together, these results suggested a higher rate of monocyte diapedesis across the vascular wall in socially isolated animals (third part of the puzzle).

TLRs signaling pathway, as an important part of the innate immune system, could orchestrate the adaptive response [45]. Both clinical and experimental reports have demonstrated that the expression of TLR4, TLR1, TLR2, and to a lesser extent TLR5 are involved in formation of atherosclerotic plaques [46]. 
Results obtained from previous evidence showed that TLR4 and its downstream pro-inflammatory cytokines increased in $\mathrm{ApoE}^{-/-}$mice [27]. In the current study, we observed that circulating monocyte of socially isolated animals have been expressed higher level of TLR4, but not TLR2, in comparison to normal group (forth part of the puzzle).

It has been demonstrated that higher expression of TLRs (TLR2, TLR4 and TLR9) can influence lipid uptake by macrophages in endothelium and increasing the formation of foam cell [47]. On the other hand, it has been reported that TLR signaling pathway may be involved in the regulation of macrophage polarization; excessive activation of TLR4 has been linked to M1 macrophage polarization rather than M2 macrophage phenotype [48]. Interestingly, it has been investigated that a higher M1/M2 ratio is associated with higher risk of atherosclerosis [48]. In this regard, Lee et al. showed that direct injection of inflammatory cytokines accelerated the formation of atherosclerotic plaque through increasing M1 macrophage polarization [48]. In the current study, we observed that macrophages are prone to express M1 phenotype rather than M2 in endothelium of socially isolated animals in comparison to normal mice (fifth part of the puzzle).

Previous studies showed that chronic stress can significantly accelerate the atherosclerosis process in ApoE knock out animals. Although, this model helped to investigate the role of high lipid profile in atherosclerotic plaque formation, this non-physiological process can not accurately reflect the human disease. In addition, it has been suggested that ApoE knock out mice are expected to have higher levels of VLDL particles, which is not typical in human atherosclerosis [7]. However, in the current study, normal animals without any genetically modification, which have been encountered with social isolation stress and high-fat diet, have shown pre-atherosclerotic lesions at late adolescence. Thus, this model might be a novel model to evaluate the pre-atherosclerosis process at physiologic and natural condition and is more similar to human plaques formation than genetically modified models.

Summary (Graphical abstract): 1) SIS over-activated the HPA-axis, and high level of corticosterone increase the expression of TLR4 on monocytes through inhibiting of NF-KB (a possible negative feedback from NF-KB to TLR4). 2) SIS increased in lipid profile components, as a risk factor for atherosclerosis process 3) SIS increased expression of adhesion molecules on vascular wall, endothelial inflammation, cardiac oxidative stress and the level of ox-LDL, 4) SIS increased expression of TLR4 on circulating monocyte, 6 ) increased in M1/M2 ratio in endothelium uptake of ox-LDL by M1 macrophage.

Overall, TLR4 might be a critical part of this puzzle to inhibit the formation of atherosclerosis. In this regard, our results showed that inhibiting TLR4 significantly decreased endothelium inflammation, M1/M2 ratio, gene expression of cell adhesion molecules, and decreased the pre-atherosclerotic lesion and intimal thickening in histopathological studies. Finally, it should be noted that SIS could not induce a significant impact on the cardiac electrical function, except for the heart rate. It is important to note that this study was used genetically normal animals instead of apolipoprotein E knock-out mice, because evaluating this topic in normal animals is far closer to natural process of atherosclerosis. 


\section{Conclusion}

This study showed that SIS could significantly increase the risk of atherosclerosis through HPA-axis, TLR4 up-regulation on circulating monocytes, increased in endothelium inflammation, increased in the gene expression of cell adhesion molecules, and finally M1 macrophage polarization in the endothelium. In addition, we observed that the formation of atherosclerotic plaques might be inhibited by blocking TLR4. In this regard, it could be concluded that TLR4 inhibitors might be a novel treatment to prevent cardio-cerebrovascular events by decreasing the risk of atherosclerosis. Finally, SIS plus high fat diet might be a novel model to evaluate the pre-atherosclerosis process at natural and physiologic condition and is more similar to human plaques formation than genetically modified models.

\section{Declarations}

\section{Funding source}

None.

\section{Acknowledgements}

None.

\section{Conflict of interest}

All authors declare that they do not have any conflict of interest.

\section{Ethics Approval and consent to participate}

Not applicable.

\section{Consent for Publication}

Not applicable.

\section{Availability of supporting data}

Not applicable.

\section{Authors' contributions}


$\mathrm{AHM}$ and $\mathrm{KR}$ have hypothesized the main concept and done the most experimental tasks and written the most part of the manuscript. SS and MT have done experimental tasks and also wrote the manuscript. AT and MR have done cell culture and gene knocking-down process and also analyzed the data and wrote the results section. AR and FP have written some parts of the manuscript including material and methods and done a few parts of experimental tasks. KK has designed figures and edited the manuscript language. NS and AK have reviewed the manuscript and re-analyzed the data for finding any errors. All experimental tasks and procedures were done under supervision of ARD.

\section{References}

1. Knox, S. S. \& Uvnäs-Moberg, K. Social isolation and cardiovascular disease: an atherosclerotic pathway? Psychoneuroendocrinology, 23, 877-890 (1998).

2. Arthur, H. M. Depression, isolation, social support, and cardiovascular disease in older adults. J. Cardiovasc. Nurs, 21, S2-S7 (2006).

3. Xia, N. \& Li, H. Loneliness, social isolation, and cardiovascular health. Antioxid. Redox Signal, 28, 837-851 (2018).

4. Valtorta, N. K., Kanaan, M., Gilbody, S., Ronzi, S. \& Hanratty, B. Loneliness and social isolation as risk factors for coronary heart disease and stroke: systematic review and meta-analysis of longitudinal observational studies. Heart, 102, 1009-1016 (2016).

5. Liu, S. W., Bin Qiao, S., Yuan, J. S. \& Liu, D. Q. Association of plasma visfatin levels with inflammation, atherosclerosis and acute coronary syndromes (ACS) in humans, Clin. Endocrinol. (Oxf), 71, 202-207 (2009).

6. Singh, U. \& Jialal, I. Oxidative stress and atherosclerosis. Pathophysiology, 13, 129-142 (2006).

7. Plump, A. S. et al. Severe hypercholesterolemia and atherosclerosis in apolipoprotein E-deficient mice created by homologous recombination in ES cells. Cell, 71.2, 343-353 (1996).

8. Inoue, N. Stress and atherosclerotic cardiovascular disease. J. Atheroscler. Thromb, 21, 391-401 (2014).

9. Shively, C. A., Register, T. C. \& Clarkson, T. B. Social stress, visceral obesity, and coronary artery atherosclerosis: product of a primate adaptation. Am. J. Primatol. Off. J. Am. Soc. Primatol, 71, 742751 (2009).

10. Haj-Mirzaian, A. et al. Lithium attenuated the depressant and anxiogenic effect of juvenile social stress through mitigating the negative impact of interlukin-1 $\beta$ and nitric oxide on hypothalamicpituitary-adrenal axis function. Neuroscience, 315 https://doi.org/10.1016/j.neuroscience.2015.12.024 (2016).

11. Veenema, A. H., Meijer, O. C., de Kloet, E. R., Koolhaas, J. M. \& Bohus, B. G. Differences in basal and stress-induced HPA regulation of wild house mice selected for high and low aggression. Horm. Behav. 43.1, 197-204 https://doi.org/10.1016/S0018-506X(02)00013-2 (2003). 
12. Ling, J. \& Kumar, R. Crosstalk between NFkB and glucocorticoid signaling: a potential target of breast cancer therapy. Cancer Lett, 322, 119-126 (2012).

13. Rao, N. A. S. et al. Coactivation of GR and NFKB alters the repertoire of their binding sites and target genes. Genome Res, 21, 1404-1416 (2011).

14. Katakami, N. Mechanism of development of atherosclerosis and cardiovascular disease in diabetes mellitus,J. Atheroscler. Thromb.(2017)RV17014.

15. Chistiakov, D. A., Melnichenko, A. A., Myasoedova, V. A., Grechko, A. V. \& Orekhov, A. N. Mechanisms of foam cell formation in atherosclerosis. J. Mol. Med, 95, 1153-1165 (2017).

16. Fang, S. et al. Irgm1 promotes M1 but not M2 macrophage polarization in atherosclerosis pathogenesis and development. Atherosclerosis, 251, 282-290 (2016).

17. de Gaetano, M., Crean, D., Barry, M. \& Belton, O. M1-and M2-type macrophage responses are predictive of adverse outcomes in human atherosclerosis. Front. Immunol, 7, 275 (2016).

18. Dou, F. et al. Anti-atherosclerotic effects of LXRa agonist through induced conversion of M1 macrophage to M2, Am. J. Transl. Res, 11, 3825 (2019).

19. Quero, L., Hanser, E., Manigold, T., Tiaden, A. N. \& Kyburz, D. TLR2 stimulation impairs antiinflammatory activity of M2-like macrophages, generating a chimeric M1/M2 phenotype. Arthritis Res. Ther, 19, 1-13 (2017).

20. Zhu, W. et al. Zoledronic acid promotes TLR-4-mediated M1 macrophage polarization in bisphosphonate-related osteonecrosis of the jaw. FASEB J, 33, 5208-5219 (2019).

21. Feng, T. T. et al. TLR-2-mediated metabolic reprogramming participates in polyene phosphatidylcholine-mediated inhibition of M1 macrophage polarization,Immunol. Res.(2020)1-11.

22. Fitzgerald, K. A. \& Kagan, J. C. Toll-like receptors and the control of immunity, Cell(2020).

23. Lin, J., Kakkar, V. \& Lu, X. Essential roles of toll-like receptors in atherosclerosis. Curr. Med. Chem, 23, 431-454 (2016).

24. Haj-Mirzaian, A. et al. Activation of cannabinoid receptors elicits antidepressant-like effects in a mouse model of social isolation stress. Brain Res. Bull, 130 https://doi.org/10.1016/j.brainresbull.2017.01.018 (2017).

25. Haj-Mirzaian, A. et al. Involvement of opioid system in behavioral despair induced by social isolation stress in mice. Biomed. Pharmacother, 109, 938-944 (2019).

26. Zhang, K. et al. Effect of Toll-like receptor 4 on depressive-like behaviors induced by chronic social defeat stress. Brain Behav, 10, e01525 (2020).

27. Gu, H. F. et al. Chronic unpredictable mild stress promotes atherosclerosis via HMGB1/TLR4mediated downregulation of PPARY/LXRa/ABCA1 in ApoE-/-Mice. Front. Physiol, 10, 165 (2019).

28. Bernberg, E., Ulleryd, M. A., Johansson, M. E. \& Bergström, G. M. L. Social disruption stress increases IL-6 levels and accelerates atherosclerosis in ApoE-/- mice. Atherosclerosis, 221, 359-365 (2012).

29. Zhang, T. et al. Chronic unpredictable stress accelerates atherosclerosis through promoting inflammation in apolipoprotein E knockout mice. Thromb. Res, 126, 386-392 (2010). 
30. Haj-Mirzaian, A. et al. Protective effect of minocycline on LPS-induced mitochondrial dysfunction and decreased seizure threshold through nitric oxide pathway. Eur. J. Pharmacol, 858, 172446 (2019).

31. Kumari, A., Singh, D. K., Dash, D. \& Singh, R. Intranasal curcumin protects against LPS-induced airway remodeling by modulating toll-like receptor-4 (TLR-4) and matrixmetalloproteinase-9 (MMP-9) expression via affecting MAP kinases in mouse model. Inflammopharmacology, 27, 731-748 (2019).

32. Wagner, M. et al. Isolation and intravenous injection of murine bone marrow derived monocytes. J. Vis. Exp, 52347 https://doi.org/10.3791/52347 (2014).

33. Haj-Mirzaian, A. et al. The potential role of very small embryonic-like stem cells in the neuroinflammation induced by social isolation stress: Introduction of a new paradigm. Brain Res. Bull, https://doi.org/10.1016/j.brainresbull.2020.07.006 (2020).

34. Qin, W. et al. Identification of functional glucocorticoid response elements in the mouse Fox01 promoter. Biochem. Biophys. Res. Commun, 450, 979-983 (2014).

35. Chuang, J. C. et al. Chronic social defeat stress disrupts regulation of lipid synthesis. J. Lipid Res, 51, 1344-1353 (2010).

36. Grant, N., Hamer, M. \& Steptoe, A. Social isolation and stress-related cardiovascular, lipid, and cortisol responses. Ann. Behav. Med, 37, 29-37 (2009).

37. Gądek-Michalska, A., Bugajski, A., Tadeusz, J., Rachwalska, P. \& Bugajski, J. Chronic social isolation in adaptation of HPA axis to heterotypic stress. Pharmacol. Reports, 69, 1213-1223 (2017).

38. Chikanza, I. C. Mechanisms of corticosteroid resistance in rheumatoid arthritis: a putative role for the corticosteroid receptor $\beta$ isoform. Ann. N. Y. Acad. Sci, 966, 39-48 (2002).

39. Sahafi, E., Peeri, M., Hosseini, M. J. \& Azarbyjani, M. A. Cardiac oxidative stress following maternal separation stress was mitigated following adolescent voluntary exercise in adult male rat. Physiol. Behav, 183, 39-45 (2018).

40. Sonei, N. et al. Mitochondrial dysfunction bridges negative affective disorders and cardiomyopathy in socially isolated rats: pros and cons of fluoxetine. World J. Biol. Psychiatry, 18, 39-53 (2017).

41. Pirillo, A., Norata, G. D. \& Catapano, A. L. LOX-1, OxLDL, and atherosclerosis, Mediators Inflamm 2013 (2013).

42. Jelic, S. \& Le Jemtel, T. H. Inflammation, oxidative stress, and the vascular endothelium in obstructive sleep apnea. Trends Cardiovasc. Med, 18, 253-260 (2008).

43. Schulz, E., Gori, T. \& Münzel, T. Oxidative stress and endothelial dysfunction in hypertension. Hypertens. Res, 34, 665-673 (2011).

44. Gerhardt, T. \& Ley, K. Monocyte trafficking across the vessel wall. Cardiovasc. Res, 107, 321-330 (2015).

45. $\mathrm{Xu}, \mathrm{X} . \mathrm{H}$. et al. Toll-like receptor-4 is expressed by macrophages in murine and human lipid-rich atherosclerotic plaques and upregulated by oxidized LDL. Circulation, 104, 3103-3108 (2001). 
46. Edfeldt, K., Swedenborg, J., Hansson, G. K. \& Yan, Z. Expression of toll-like receptors in human atherosclerotic lesions: a possible pathway for plaque activation. Circulation, 105, 1158-1161 (2002).

47. Cole, J. E., Georgiou, E. \& Monaco, C. The expression and functions of toll-like receptors in atherosclerosis, Mediators Inflamm 2010 (2010).

48. Lee, S. G. et al. Macrophage polarization and acceleration of atherosclerotic plaques in a swine model. PLoS One, 13, e0193005 (2018).

Figures 

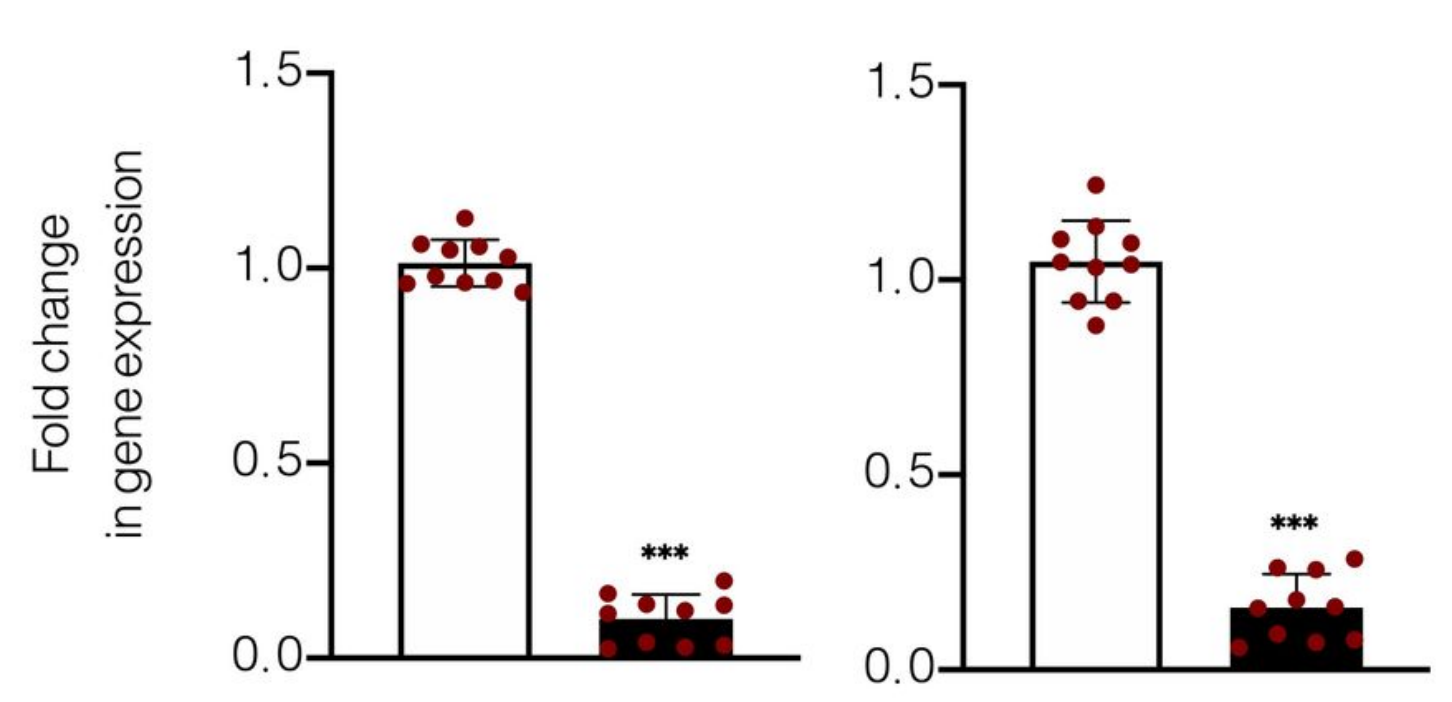

B

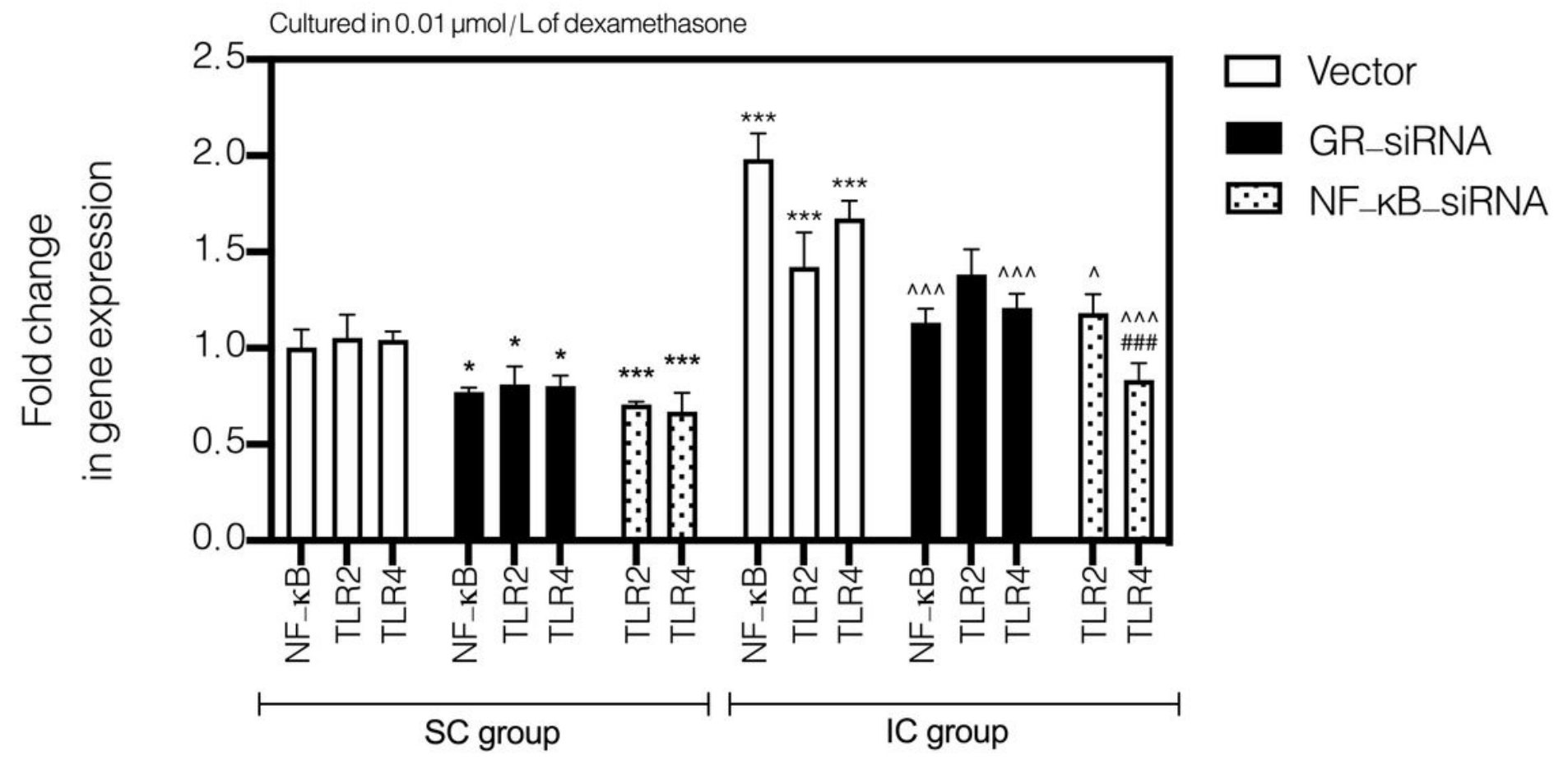

Figure 1

Fold change in gene expression of TLR2, TLR4, and NF-KB in GR- and NF-KB-knock-down cultured monocytes derived from bone marrow of socially isolated animals. Values are expressed as the mean \pm S.E.M. ( $\mathrm{n}=10$ in each group) results were analyzed using One-Way ANOVA followed by Tukey's post hoc test. ${ }^{\star \star \star} \mathrm{P}<0.001$ compared to social condition group treated with vector. $\# \# \# \mathrm{P}<0.001$ compared to 
isolated condition group treated with GR-siRNA, $\wedge P<0.05$ and $\wedge \wedge \wedge P<0.001$ compared to social isolation group treated with vector.

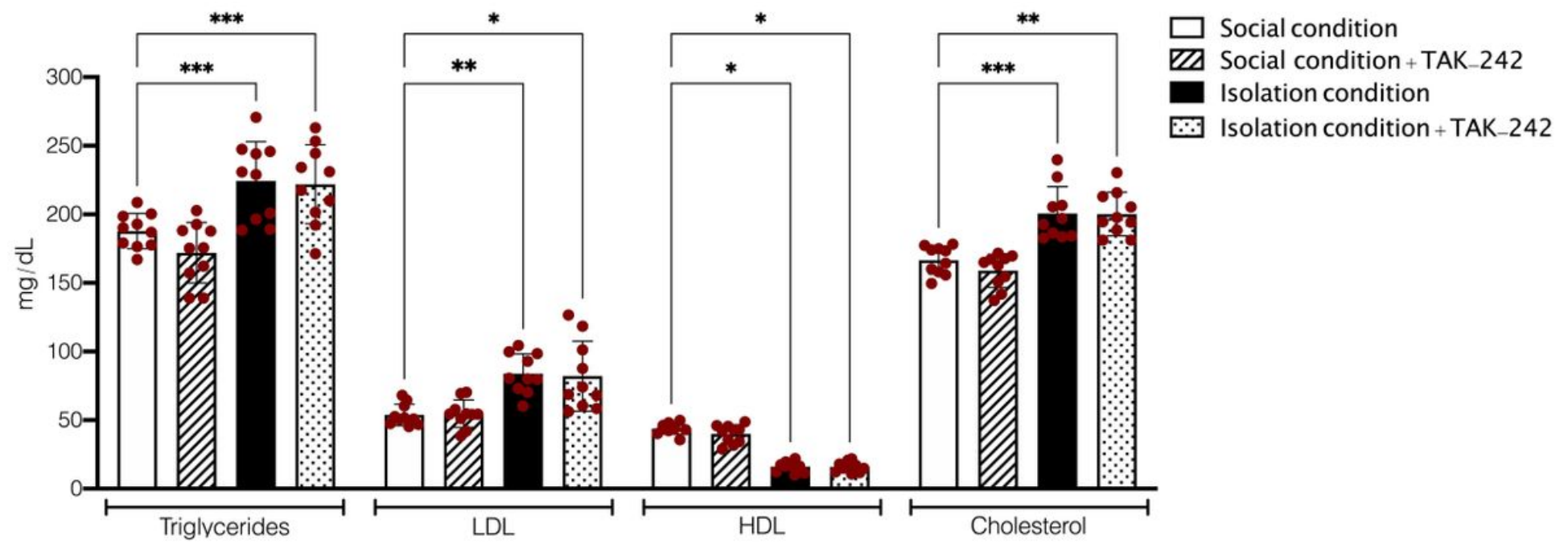

Figure 2

Results of plasma concentrations of lipid profile components in socially isolated and normal animals. Values are expressed as the mean \pm S.E.M. ( $n=10$ in each group) results were analyzed using One-Way ANOVA followed by Tukey's post hoc test. ***P $<0.001$ compared to SC animals.

Social condition
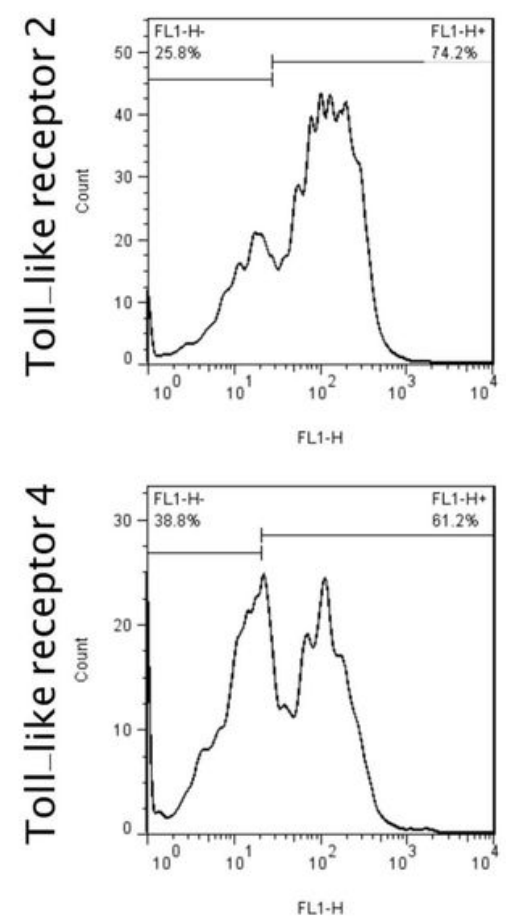

Isolation condition
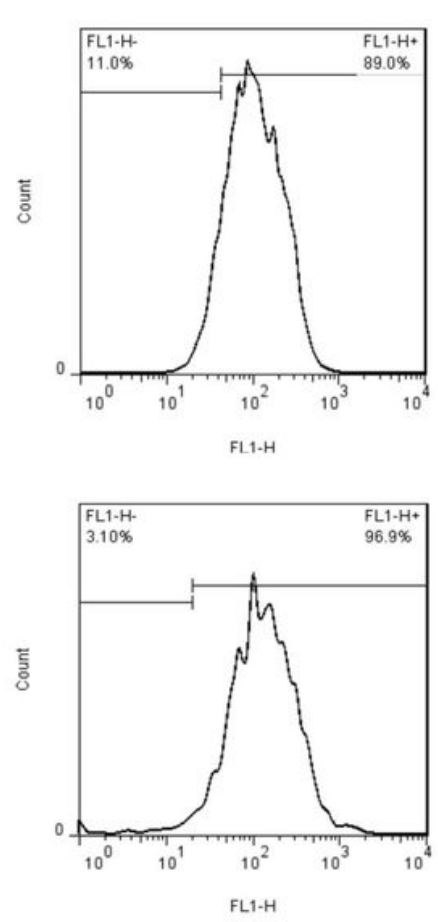
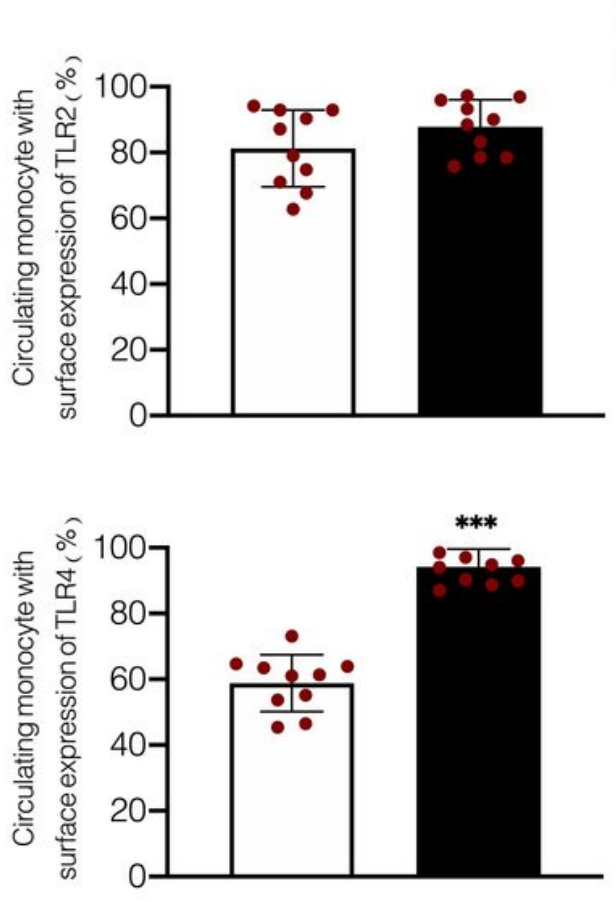

Figure 3

The expression of TLR-2 and 4 on circulating monocytes in both normal and SI animals. Values are expressed as the mean \pm S.E.M. ( $n=10$ in each group) results were analyzed using One-Way ANOVA followed by Tukey's post hoc test. ${ }^{*} * \mathrm{P}<0.001$ compared to SC animals. 


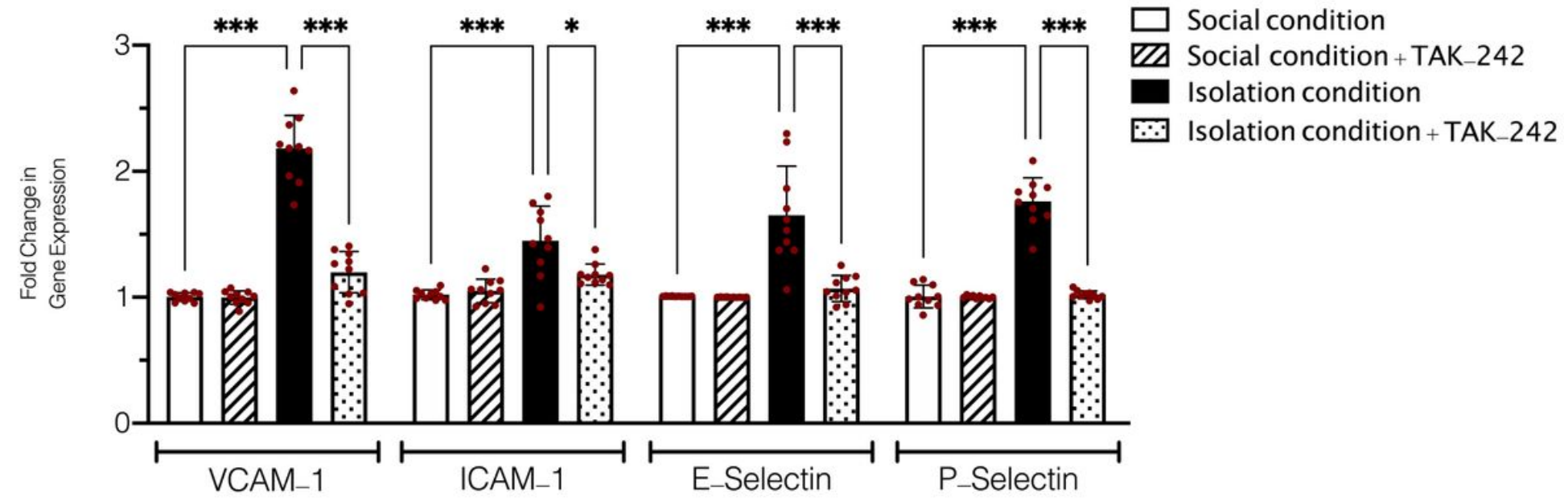

Figure 4

Fold change in gene expression of VCAM-1, ICAM-1, E-selectin, P-selectin receptors among SC, IC, and IC animals treated with TAK-242 in entire aorta samples. Values are expressed as the mean \pm S.E.M. $(n=10$ in each group) results were analyzed using One-Way ANOVA followed by Tukey's post hoc test. * $P<0.05$ and ${ }^{* *} \mathrm{P}<0.001$ compared to SC animals. \#P<0.05 and \#\#\#P<0.001 compared to IC animals.
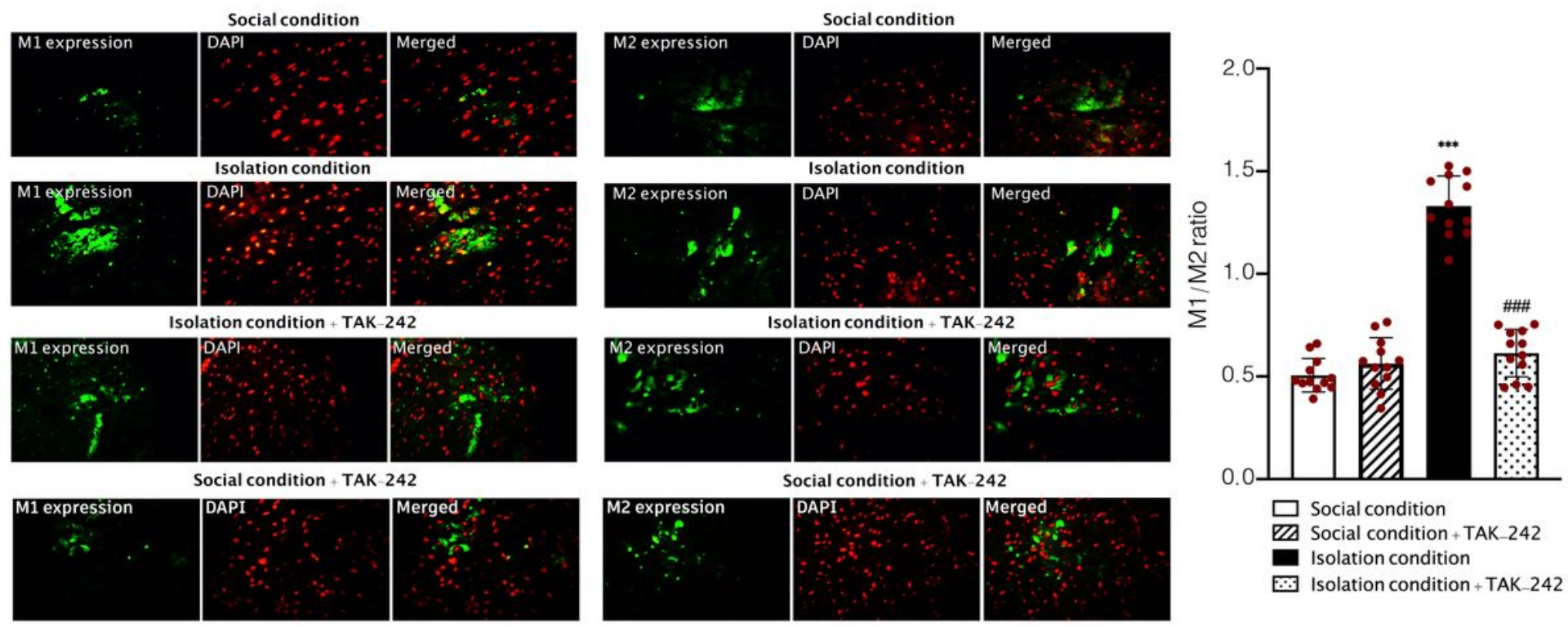

\section{Figure 5}

The expression of M1 and M2 biomarkers in entire aorta samples in SC and IC animals. Values are expressed as the mean \pm S.E.M. ( $n=12$ in each group) results were analyzed using One-Way ANOVA followed by Tukey's post hoc test. ${ }^{\star * \star} \mathrm{P}<0.001$ compared to SC animals. \#\#\#P<0.001 compared to IC animals. 

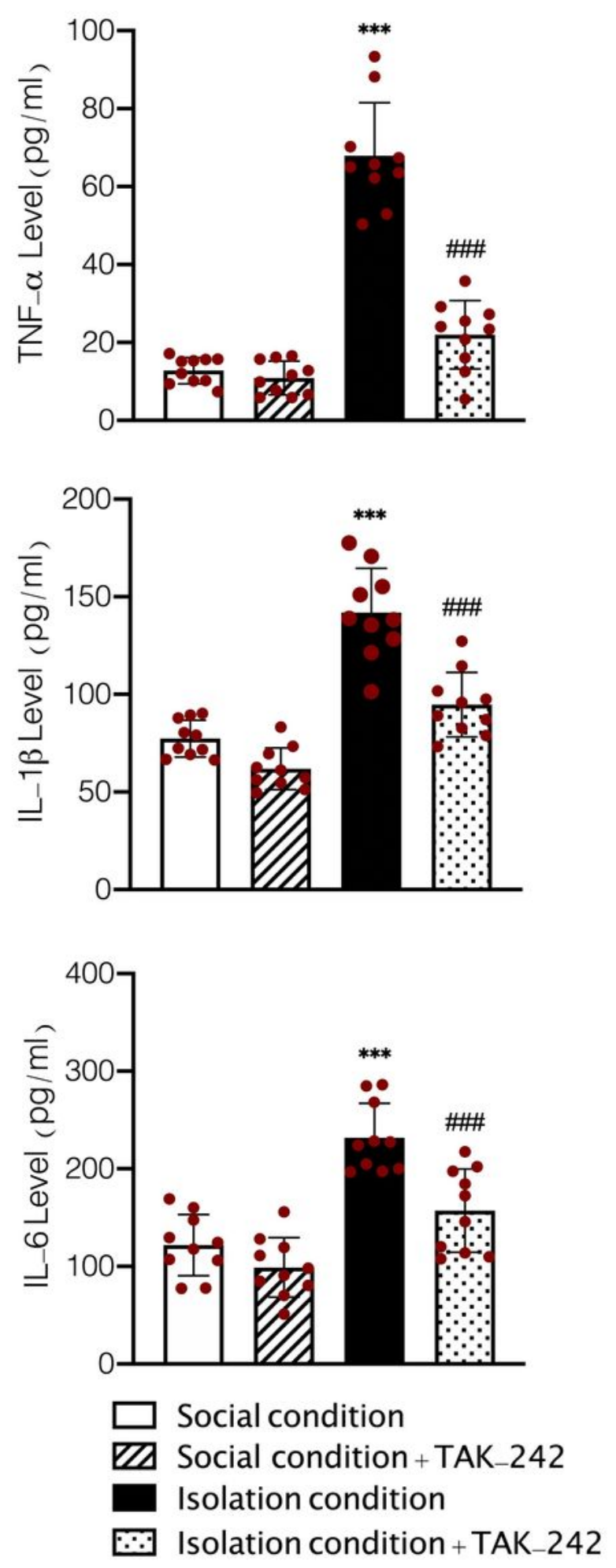

Figure 6

Effects of SIS on concentration of TNF-a (A), IL-1ß (B), and IL-6 (C) in entire aorta samples. Values are expressed as the mean \pm S.E.M. ( $n=10$ in each group) results were analyzed using One-Way ANOVA followed by Tukey's post hoc test. ${ }^{* \star *} \mathrm{P}<0.001$ compared to $\mathrm{SC}$ animals and \#\#\#P<0.001 compared to IC animals. 


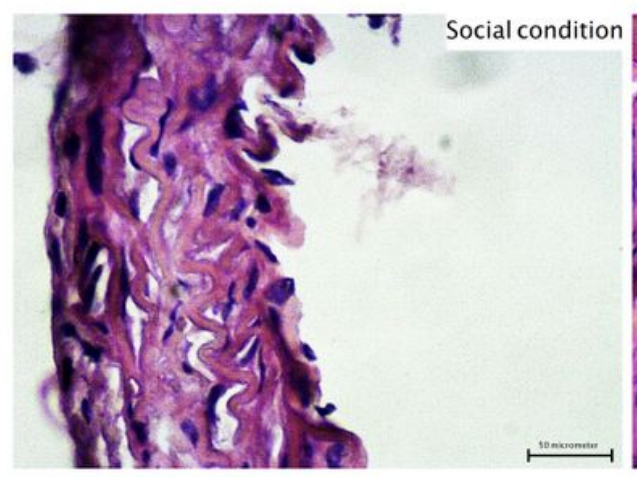

* Isolation condition + TAK_242

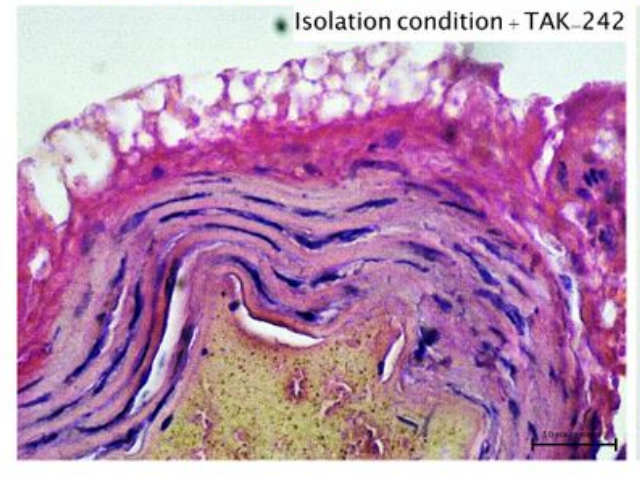

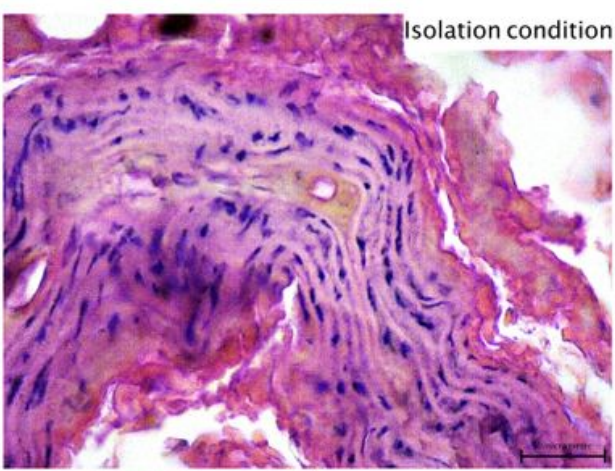

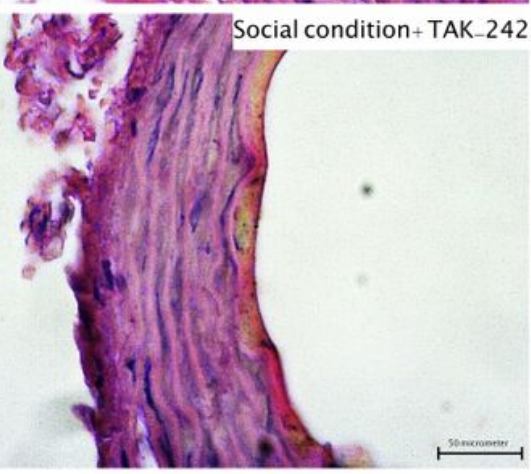

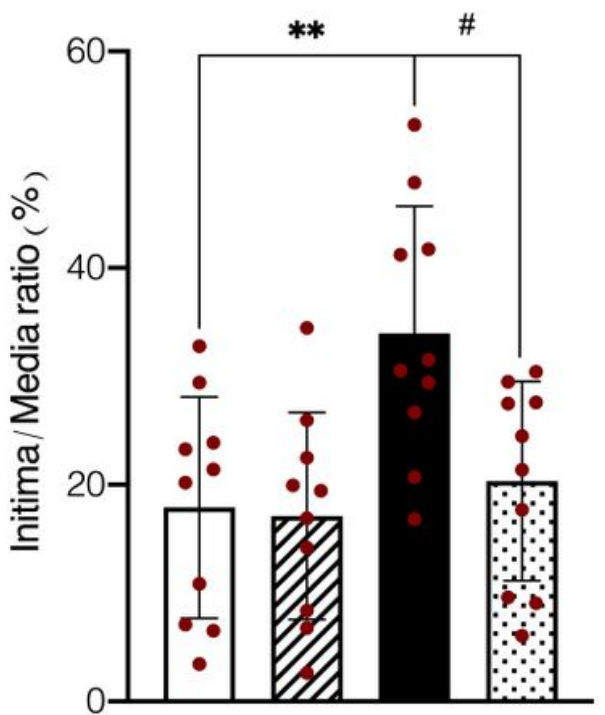

Social condition

Z2 Social condition+TAK-242

Isolation condition

$\because$ Isolation condition + TAK_242

Figure 7

Pre-atherosclerotic intimal lesions and intimal/media thickness (ratio) assessment in IC and IC animals treated with TAK-242 in comparison to SC animals (40x magnification). Values are expressed as the mean \pm S.E.M. ( $n=10$ in each group) results were analyzed using One-Way ANOVA followed by Tukey's post hoc test. ${ }^{*} \mathrm{P}<0.01$ compared to $\mathrm{SC}$ animals. $\# \mathrm{P}<0.01$ compared to IC animals. 